\title{
Rms and Power Measurements: A Wavelet Packet Transform Approach
}

\author{
Non-member Effrina Yanti Hamid (Osaka University) \\ Student Member Nobuaki Yokoyama (Osaka University) \\ Member $\quad$ Zen-Ichiro Kawasaki (Osaka University)
}

\begin{abstract}
This study provides the theoretical basis for the use of wavelet packet transform (WPT) approach for root mean square (rms) and power/energy measurements. The proposed approach can simultaneously measure the distribution of the rms and power with respect to individual frequency bands directly from the wavelet transform coefficients (WTCs) associated with concurrent voltage current pair. Their dependent quantities such as power factor and total harmonic band distortion can be calculated as well. Uniform frequency bands are yielded from the WPT decomposition process of power system waveforms and can be used for identification of harmonic components. The frequency bands also retain both the time and frequency relationship of the original waveforms, which is one of the major benefits provided by this approach. The approach is evaluated by its application to both analytical and actual power system waveforms.
\end{abstract}

Keywords: Power, Root mean square (Rms), Harmonics, Wavelet packet transform.

\section{Introduction}

The traditional methods for rms of voltage and current, and power/energy measurements in electric power system have been performed in both the time domain and in the frequency domain using the well-known Fourier transform approach. The time domain approach is the most accurate and efficient when total rms and total power as well as power factor are concerned. The frequency domain approach permits to measure the rms of individual frequency components and, hence, it allows to provide the rms of individual bands and the determination of harmonic distortion, but it suffers from the requirement of stationary waveform conditions, where the load waveforms are always dynamic in nature, and the loss of temporal insight ${ }^{(1)}$.

Wavelet has proven a powerful signal processing tool for transient analysis of power system waveforms ${ }^{(2)(3)}$. However, there has not been much work on applying wavelet transform to rms and power/energy measurements. The wavelet analysis for $\mathrm{rms}$ and power measurements using the conventional discrete wavelet transform (DWT) approach has first been introduced in the literature ${ }^{(4)}$. The advantage of using the wavelet transform is that it preserves both the time and frequency relationship associated with the resulting rms and power values. However, the DWT technique is not suitable for harmonic analysis, because the resulted frequency bands do not have the same width and the results do not give easy insight in the time behavior of the harmonics ${ }^{(5)}$. For instance, at a lower level of decomposition the band becomes wider and covers more harmonic components than the band at higher level. Consequently, the resulted rms and power with respect to individual bands may contain several harmonic components. The capability to provide uniform bands are moreover important for harmonic identification purposes. In fact, harmonic distortion is most frequently observed and it is most necessary to be identified in order to know the sources and thus to eliminate its effects ${ }^{(6)(7)}$. To overcome the above limitation the WPT-based approach is proposed. The approach decomposes the given power system waveforms into a set of harmonic bands with the same frequency width, and each of the bands represents that part of the original instantaneous rms and power occurring at that particular time and in that particular frequency band. This means that the approach provides the distribution of $\mathrm{rms}$ and power/energy with respect to individual frequency bands of wavelet analysis.

This study starts with a brief review of wavelet packet theory. Then, it is followed by the representation of rms and power measurements in wavelet domain. Their dependent quantities such as power factor and total harmonic band distortion are also calculated. Last, this WPT approach is evaluated on analytical and actual voltage-current waveforms, and the results are further compared to those obtained from the time domain and frequency domain methods.

\section{Wavelet Packet Transform}

2.1 Theory While detail mathematical background of wavelet packet transform (WPT) can be found in ${ }^{(8)-(10)}$, a brief summary is given in this section. The WPT is a direct expansion of the conventional DWT algorithm. In the WPT, both the approximation space and the detail space are decomposed to derive new lower resolution approximation spaces plus detail spaces. Let $\phi(t)$ and $\psi(t)$ be the scaling function and the corresponding mother wavelet function in 
the conventional DWT and define $\psi^{0}(t)=\phi(t)$, and $\psi^{1}(t)=\psi(t)$. Using the well-known "two-scale equations," we can construct the wavelet basis as follows:

$$
\begin{aligned}
\psi_{j, k}^{2 i}(t) & =\frac{1}{\sqrt{2^{j}}} \psi^{2 i}\left(\frac{2^{j} k-t}{2^{j}}\right)=\sum_{n} h(n) \psi_{j-1,2 k-n}^{i}(t) \cdot(1) \\
\psi_{j, k}^{2 i+1}(t) & =\frac{1}{\sqrt{2^{j}}} \psi^{2 i+1}\left(\frac{2^{j} k-t}{2^{j}}\right)=\sum_{n} g(n) \psi_{j-1,2 k-n}^{i}(t)(2)
\end{aligned}
$$

where $i$ is the node or band number, $j$ is the decomposition level, and $h(n)$ and $g(n)=(-1)^{1-n} h(1-n)$ are a pair of quadrature mirror filters (QMFs). The wavelet transform coefficients (WTCs) of a given function $f(t)$ at the $j$ th level and $k$ th point are computed via the following recursion relations:

$$
\begin{aligned}
d_{j}^{2 i}(k) & =\int f(t) \psi_{j, k}^{2 i}(t) d t=\sum_{n} h(n) d_{j-1}^{i}(2 k-n) \\
d_{j}^{2 i+1}(k) & =\int f(t) \psi_{j, k}^{2 i+1}(t) d t=\sum_{n} g(n) d_{j-1}^{i}(2 k-n) .
\end{aligned}
$$

Since the wavelet basis in (1) and (2) are orthogonal, they satisfy the following properties ${ }^{(8)-(10)}$ :

$$
\begin{array}{rlrl}
\int \psi_{j, k}^{p}(t) d t & =0 & & p=\text { integer } \\
\int \psi_{j, k}^{p}(t) \psi_{j, k}^{q}(t) d t & =1 & & p=q \\
\int \psi_{j, k}^{p}(t) \psi_{j, k}^{q}(t) d t & =0 & & p \neq q \\
\int\left(\phi_{j, k}(t)\right)^{2} d t & =1 & \\
\int \phi_{j, k}(t) \psi_{j, k}^{p}(t) d t & =0 & &
\end{array}
$$

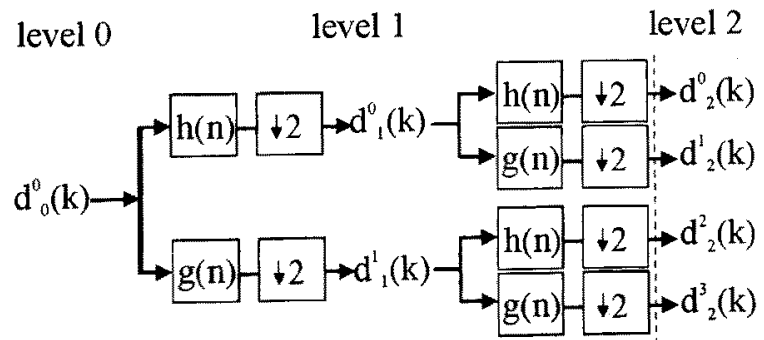

Decomposition

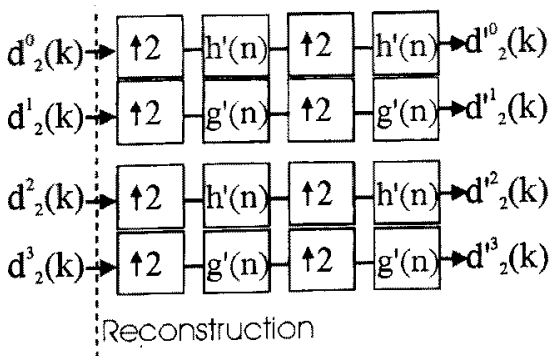

Fig. 1. Top figure is wavelet packet decomposition with successive filterings and downsamplings, and bottom figure is signal reconstruction for each band from the resulted wavelet transform coefficients.
2.2 Decomposition Wavelet packet decomposition is depicted in Fig. 1. Let the original waveform has $2^{N}$ sampling points. The WTC at the $j$ th level, $k$ th point and $2 i$ th node is $d_{j}^{2 i}(k)$, where $j=0,1, \ldots, N$ and $i=0,1, \ldots, 2^{j-1}-1$. This WTC is obtained by convolving the sequence $d_{j-1}^{i}(k)$ with low-pass filter $h(n)$, and then downsampling by a factor of two. Similarly, the WTC at $(2 i+1)$ th node, that is $d_{j}^{2 i+1}(k)$, is obtained by convolving the sequence $d_{j-1}^{i}(k)$ with high-pass filter $g(n)$ and downsampling by a factor of two. Number of nodes or bands at $j$ th level is $2^{j}$, and the node at level 0 is the original waveform.

The time resolution of $d_{j}^{2 i}(k)$ (or $d_{j}^{2 i+1}(k)$ ) is half that of $d_{j-1}^{2 i}(k)$ (or $d_{j-1}^{2 i+1}(k)$ ), due to the downsampling. As a result, if $d_{j-1}^{2 i}(k)$ (or $d_{j-1}^{2 i+1}(k)$ ) has $2^{N-j+1}$ points $\left(k=0,1, \ldots, 2^{N-j+1}-1\right)$ for the entire observation period, then $d_{j}^{2 i}(k)$ (or $d_{j}^{2 i+1}(k)$ ) will have $2^{N-j}$ points $\left(k=0,1, \ldots, 2^{N-j}-1\right)$ for the same observation period. Every band at $j$ th level has $2^{N-j}$ points or WTCs.

The implementation of signal reconstruction is fairly straightforward. The waveform reconstruction of each band can be performed using the resulted WTCs from the decomposition process, and the procedure has a reversal process which includes upsampling by a factor of two and filtering as seen in Fig. 1. Mathematically, the reconstructed waveform coefficients of each band can be written as

$$
\begin{aligned}
d_{j-1}^{2 i}(k) & =\sum_{n} h(n) d_{j}^{2 i}(2 n-k) \\
d_{j-1}^{2 i+1}(k) & =\sum_{n} g(n) d_{j}^{2 i+1}(2 n-k)
\end{aligned}
$$

2.3 Wavelet Filter The selection of which wavelet to use for the measurement is not an easy task. Therefore, instead of creating algorithms to select appropriate wavelets (which surely adds complexity to the main problem), we utilize one type of wavelet in the whole course of measurements for all voltage current pair waveforms. Since accurate measurements are con-
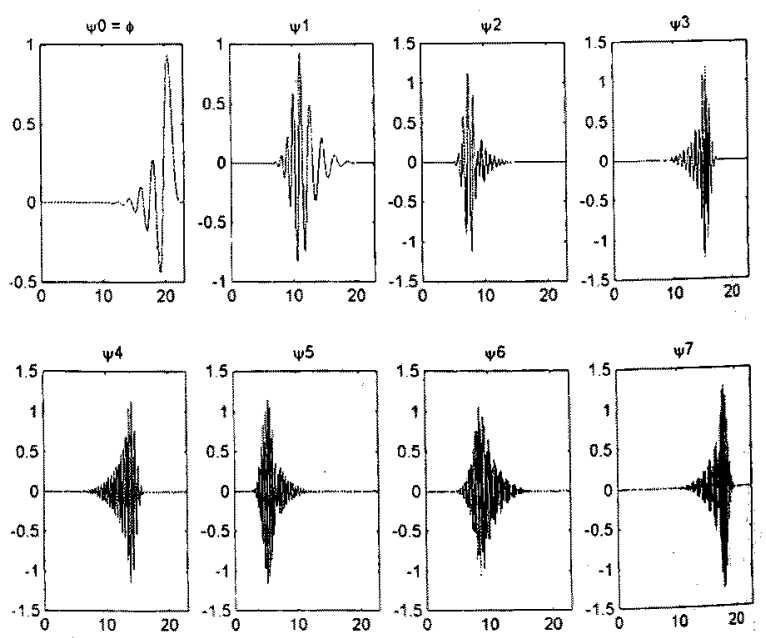

Fig. 2. Wavelet packet basis computed with the Vaidyanathan filter up to level 3. They are ordered from low to high frequencies. 

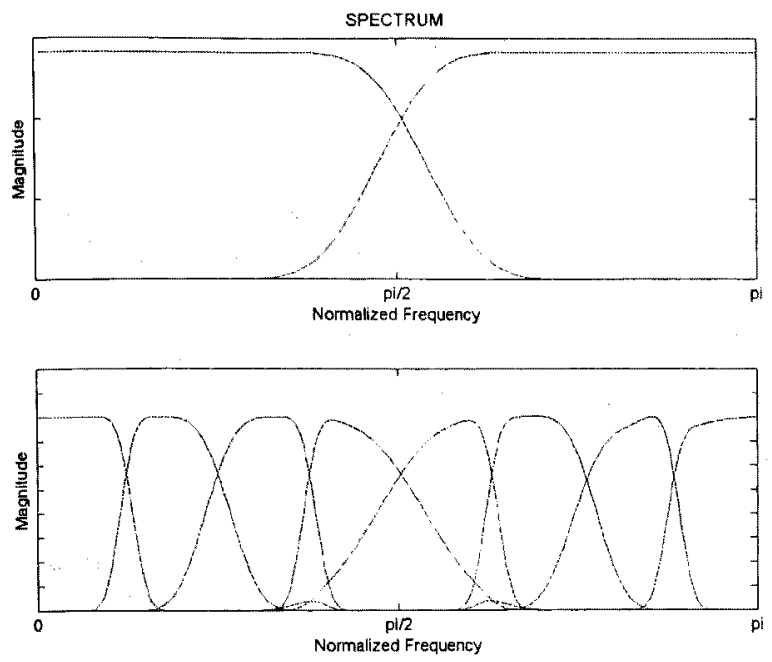

Fig. 3. Frequency responses of the Vaidyanathan QMFs and 3-scale wavelet filters.

cerned, the filters which have good frequency separation between low-pass and high-pass spectrum are generally required ${ }^{(4)}$. The Vaidyanathan filter with 24 coefficients is used in the analysis because the frequency responds of this filter has good frequency separation between lowpass and high-pass spectrum ${ }^{(8)}$. Figure 2 shows the wavelet functions computed with the Vaidyanathan filter, and Fig. 3 shows the frequency responses of the associated QMFs and 3-scale wavelet filters, respectively.

\section{Wavelet Representation of Rms and Power}

3.1 Wavelet Transform of Voltage and Current Let $v(t)$ and $i(t)$ be respectively the analog voltage and current waveforms in electric power system during the observation period $T$. In practice, the analog waveforms are digitized. Here, $v(n)$ and $i(n)$ will be the digitized waveforms of $v(t)$ and $i(t)$, respectively, with $n=0,1, \ldots, 2^{N}-1$. In the wavelet theory, any time domain waveform can be expanded as a linear combination of weighted sums of wavelet basis functions ${ }^{(8)-(10)}$. Hence, $v(t)$ can be represented as

$$
\begin{aligned}
v(t) & =\sum_{i=0}^{2^{j-1}-1} \sum_{k=0}^{2^{N-j}-1} d_{j}^{2 i}(k) \psi_{j, k}^{2 i}(t) \\
& +\sum_{i=0}^{2^{j-1}-1} \sum_{k=0}^{2^{N-j}-1} d_{j}^{2 i+1}(k) \psi_{j, k}^{2 i+1}(t) \\
& =\sum_{k=0}^{2^{N-j}-1} d_{j}^{0}(k) \phi_{j, k}(t)+\sum_{i=1}^{2^{j}-1} \sum_{k=0}^{2^{N-j}-1} d_{j}^{i}(k) \psi_{j, k}^{i}(t)
\end{aligned}
$$

where $d_{j}^{0}(k)$ is the scaling function coefficients (or WTCs at $i=0)$ and $d_{j}^{i}(k)(i>0)$ is the WTCs of $v(n)$. Similarly for the current $i(t)$, it can be represented as

$$
\begin{aligned}
i(t) & =\sum_{i=0}^{2^{j-1}-1} \sum_{k=0}^{2^{N-j}-1} d_{j}^{* 2 i}(k) \psi_{j, k}^{2 i}(t) \\
& +\sum_{i=0}^{2^{j-1}} \sum_{k=0}^{2^{N-j}-1} d_{j}^{* 2 i+1}(k) \psi_{j, k}^{2 i+1}(t) \\
& =\sum_{k=0}^{2^{N-j}-1} d_{j}^{* 0}(k) \phi_{j, k}(t)+\sum_{i=1}^{2^{j}-1} \sum_{k=0}^{2^{N-j}-1} d_{j}^{* i}(k) \psi_{j, k}^{i}(t) .(9)
\end{aligned}
$$

where $d_{j}^{* 0}(k)$ and $d_{j}^{* i}(k)(i>0)$ are respectively the scaling function coefficients (or WTCs at $i=0$ ) and the WTCs of $i(n)$. The node $i=0$ is the lowest band of the original waveform and includes the fundamental frequency component. The nodes $i>0$ include the waveforms of successively higher frequency bands.

3.2 Wavelet Representation of Rms Rms of voltage $\left(V_{r m s}\right)$ and current $\left(I_{r m s}\right)$ can be derived from the WTCs in (8) and (9) at a certain level $j$ as follows

$$
\begin{aligned}
\int v(t)^{2} d t & =\int\left[\sum_{k=0}^{2} d_{j}^{0}(k) \phi_{j, k}(t)+\sum_{i=1}^{2^{j}-1} \sum_{k=0}^{N-j} d_{j}^{i}(k) \psi_{j, k}^{i}(t)\right]^{2} d t \\
& =\int\left[\sum_{k=0}^{2^{N-j}} d_{j}^{0}(k) \phi_{j, k}(t)\right]^{2} d t \\
& +\int\left[\sum_{i=1}^{2^{j}-1} \sum_{k=0}^{N-j} d_{j}^{i}(k) \psi_{j, k}^{i}(t)\right]^{2} d t \\
& +2 \int\left[\sum_{i=1}^{2^{j}-1} \sum_{k=0}^{N-j} d_{j}^{0}(k) d_{j}^{i}(k) \phi_{j, k}(t) \psi_{j, k}^{i}(t)\right] d t \\
& =\sum_{k=0}^{N-j}{ }^{2}\left(d_{j}^{0}(k)\right)^{2} \int\left(\phi_{j, k}(t)\right)^{2} d t \\
& +\sum_{i=1}^{2^{j}-1} \sum_{k=0}^{N-j}\left(d_{j}^{i}(k)\right)^{2} \int\left(\psi_{j, k}^{i}(t)\right)^{2} d t \\
& +2 \sum_{i=1}^{2^{j}-1} \sum_{k=0}^{N-j} d_{j}^{0}(k) d_{j}^{i}(k) \int \phi_{j, k}(t) \psi_{j, k}^{i}(t) d t
\end{aligned}
$$

from the wavelet properties in (5), it is known that $\int\left(\phi_{j, k}(t)\right)^{2} d t=1, \int\left(\psi_{j, k}^{i}(t)\right)^{2} d t=1$, and $\int \phi_{j, k}(t) \psi_{j, k}^{i}(t) d t=0$. Hence, this equation becomes

$$
\int v(t)^{2} d t=\sum_{i=0}^{2^{j}-1} \sum_{k=0}^{2^{N-j}-1}\left(d_{j}^{i}(k)\right)^{2} .
$$

The (total) rms of $v(t)$, based on IEEE Std. 100-88, becomes

$$
\begin{aligned}
V_{r m s} & =\sqrt{\frac{1}{T} \int_{0}^{T} v(t)^{2} d t} \simeq \sqrt{\frac{1}{2^{N}} \sum_{n=0}^{2^{N}-1} v(n)^{2}} \cdots \cdots \\
& \simeq \sqrt{\frac{1}{2^{N}} \sum_{i=0}^{2^{j}-12^{N}} \sum_{k=0}^{N-1}\left(d_{j}^{i}(k)\right)^{2}}=\sqrt{\sum_{i=0}^{2^{j}-1}\left(V_{j}^{i}\right)^{2}, \cdots}
\end{aligned}
$$

where

$$
V_{j}^{i}=\sqrt{\frac{1}{2^{N}} \sum_{k=0}^{2^{N-j}-1}\left(d_{j}^{i}(k)\right)^{2}} .
$$

Here, $V_{j}^{i}$ is the rms value of frequency band at node $i$.

The (total) rms of current $i(t)$ can be derived in similar manner, and it can be written as 


$$
\begin{aligned}
I_{T^{\prime}, n s} & =\sqrt{\frac{1}{T} \int_{0}^{T} i(t)^{2} d t} \simeq \sqrt{\frac{1}{2^{N}} \sum_{n=0}^{2^{N}-1} i(n)^{2}} \ldots \ldots \cdots \\
& \simeq \sqrt{\frac{1}{2^{N}} \sum_{i=0}^{2^{j}-1} \sum_{k=0}^{2^{N-j}-1}\left(d_{j}^{* i}(k)\right)^{2}}=\sqrt{\sum_{i=0}^{2^{j}-1}\left(I_{j}^{i}\right)^{2}, \cdots}
\end{aligned}
$$

where

$$
I_{j}^{i}=\sqrt{\frac{1}{2^{N}} \sum_{k=0}^{2^{N-j}-1}\left(d_{j}^{* i}(k)\right)^{2} .}
$$

Here, $I_{j}^{i}$ is the rms value of frequency band at node $i$.

3.3 Wavelet Representation of Power One usually uses wavelet analysis focused on individual waveforms ${ }^{(1)(3)(7)}$. However, in the case of active power/energy measurement the concurrent voltage and current waveforms are used together. If the WTCs of $v(t)$ and $i(t)$ are scaled by the same wavelet packet function $\psi(t)$, the energy is described as follows

$$
\begin{aligned}
& \int v(t) i(t) d t=\int\left[\sum_{k=0}^{2^{N}-j-1} d_{j}^{0}(k) \phi_{j, k}(t)+\sum_{i=1}^{2^{j}-1} \sum_{k=0}^{2^{N-j}-1} d_{j}^{i}(k) \psi_{j, k}^{i}(t)\right]
\end{aligned}
$$

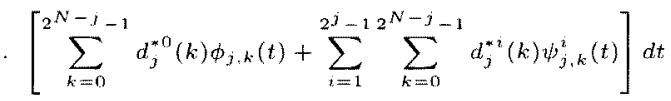

$$
\begin{aligned}
& \left.=\int^{2^{N}-j} \sum_{k=0}^{-1} d_{j}^{0}(k) d_{j}^{* 0}(k) \phi_{j, k}(t)\right)^{2} d t \\
& +\int \sum_{i=1}^{2^{j}-1} \sum_{k=0}^{N-j}-1 d_{j}^{i}(k) d_{j}^{* i}(k)\left(\psi_{j, k}^{i}(t)\right)^{2} d t \\
& +\int \sum_{i=1}^{2^{j}-1} \sum_{k=0}^{2^{N}-j} d_{j}^{n}(k) d_{j}^{* i}(k) \phi_{j, k}(t) \psi_{j, k}^{i}(t) d t \\
& +\int \sum_{i=1}^{2^{j} \sum_{-1}} \sum_{k=0}^{N-j} d_{j}^{* 0}(k) d_{j}^{i}(k) \phi_{j, k}(t) \psi_{j, k}^{i}(t) d t \\
& =\sum_{k=0}^{N-j} d_{j}(k)^{0} d_{j}^{* 0}(k) \int\left(\phi_{j, k}(t)\right)^{2} d t \\
& +\sum_{i=1}^{{ }^{2} j} \sum_{k=0}^{1}{ }^{N-j} d_{j}^{i}(k) d_{j}^{* i}(k) \int\left(\psi_{j, k}^{i}(t)\right)^{2} d t \\
& +\sum_{i=1}^{2^{j}-1} \sum_{k=0}^{2^{N-j-1}} d_{j}^{0}(k) d_{j}^{* i}(k) \int \phi_{j, k}(t) \psi_{j, k}^{i}(t) d t \\
& +\sum_{i=1}^{2^{i}-1} \sum_{k=0}^{2^{N-j}-1} d_{j}^{* 0}(k) d_{j}^{i}(k) \int \phi_{j, k}(t) \psi_{j, k}^{i}(t) d t .
\end{aligned}
$$

From the wavelet properties in (5), it is known that $\int\left(\phi_{j, k}(t)\right)^{2} d t=1, \int\left(\psi_{j, k}^{i}(t)\right)^{2} d t=1$, and $\int \phi_{j, k}(t) \psi_{j, k}^{i}(t) d t=0$. Then, the above equation becomes

$$
\int v(t) i(t) d t=\sum_{i=0}^{2^{j}-1} \sum_{k=0}^{2^{N-j}-1} d_{j}^{i}(k) d_{j}^{* i}(k) .
$$

The (total) active power $P$ (IEEE Std. 100-88) in wavelet domain using (15) becomes

$$
\begin{aligned}
P & =\frac{1}{T} \int_{0}^{T} v(t) i(t) d t \simeq \frac{1}{2^{N}} \sum_{n=0}^{2^{N}-1} v(n) i(n) \cdots \cdots \\
& \simeq \frac{1}{2^{N}} \sum_{i=0}^{2^{i}-1} \sum_{k=0}^{2^{N-j}-1} d_{j}^{i}(k) d_{j}^{* i}(k)=\sum_{i=0}^{2^{j}-1} P_{j}^{i}, \cdots
\end{aligned}
$$

where

$$
P_{j}^{i}=\frac{1}{2^{N}} \sum_{k=0}^{2^{N-j}-1} d_{j}^{i}(k) d_{j}^{* i}(k)
$$

Here, $P_{j}^{i}$ is the power value of frequency band at node $i$.

3.4 Power Factor and Total Harmonic Band Distortion In addition to the rms and power measurements, it is also possible to calculate the power factor and total harmonic band distortion (THBD) from their WTCs. It is noted that wavelet packet transform can not extract any single frequency component. Rather, the transform brings a frequency band around the frequency of interest. The word 'band' in the THBD refers to this issue. The power factor is defined as the ratio of the active power to the apparent power. It can be calculated using (12), (14), and (17):

$$
p . f .=\frac{P}{V_{r m s} \cdot I_{r m s}}=\frac{\sum_{i=0}^{2^{j}-1} P_{j}^{i}}{\sqrt{\sum_{i=0}^{2^{j}-1}\left(V_{j}^{i}\right)^{2}} \cdot \sqrt{\sum_{i=0}^{2^{j}-1}\left(I_{j}^{i}\right)^{2}}} .
$$

The THBD is defined as the ratio of the rms value of the harmonic bands at nodes $i>0$ to the rms value of the original distorted waveform. The THBD of voltage and current is respectively:

$$
\begin{aligned}
& T H B D_{v}=\frac{1}{V_{r m s}} \sqrt{\sum_{i=1}^{2^{j}-1}\left(V_{j}^{i}\right)^{2}} \\
& T H B D_{i}=\frac{1}{I_{r m s}} \sqrt{\sum_{i=1}^{2^{j}-1}\left(I_{j}^{i}\right)^{2} .}
\end{aligned}
$$

\section{Evaluation and Results}

In order to evaluate the accuracy of rms and power measurements of the proposed approach, two examples will be analyzed. The first one is software generated waveforms and the last one is actual power system waveforms. In both examples, each voltage or current waveform has eight $60 \mathrm{~Hz}$ fundamental cycles with the sampling frequency used is $7680 \mathrm{~Hz}$ or 128 points per $60 \mathrm{~Hz}$ cycle. This means that the original waveform has a length of $1024(N=10)$ points during the observation period $T=8 / 60 \mathrm{~s}$. Both original waveforms are first decomposed as described in section 2 . Only the WTCs at level $j=5$ are used to calculate rms and power because each frequency band at level 5 completely covers a respective odd harmonic component. Figure 4 illustrates the wavelet packet decomposition up to 5th level together with the frequency ranges of each band and the odd harmonic components included in each band. Level 5 has $2^{5}=32$ bands (or nodes) and each band has $2^{10-5}=32$ WTCs. Further, the original waveform has a bandwidth of $3840 \mathrm{~Hz}$ (Nyquist frequency), and each band has a bandwidth of $3840 / 32=120 \mathrm{~Hz}$.

4.1 Simulated Voltage and Current These voltage and current waveforms are periodic under steady state condition as shown in Fig. 5. The waveforms contain first, third, fifth, seventh, ninth, eleventh, 


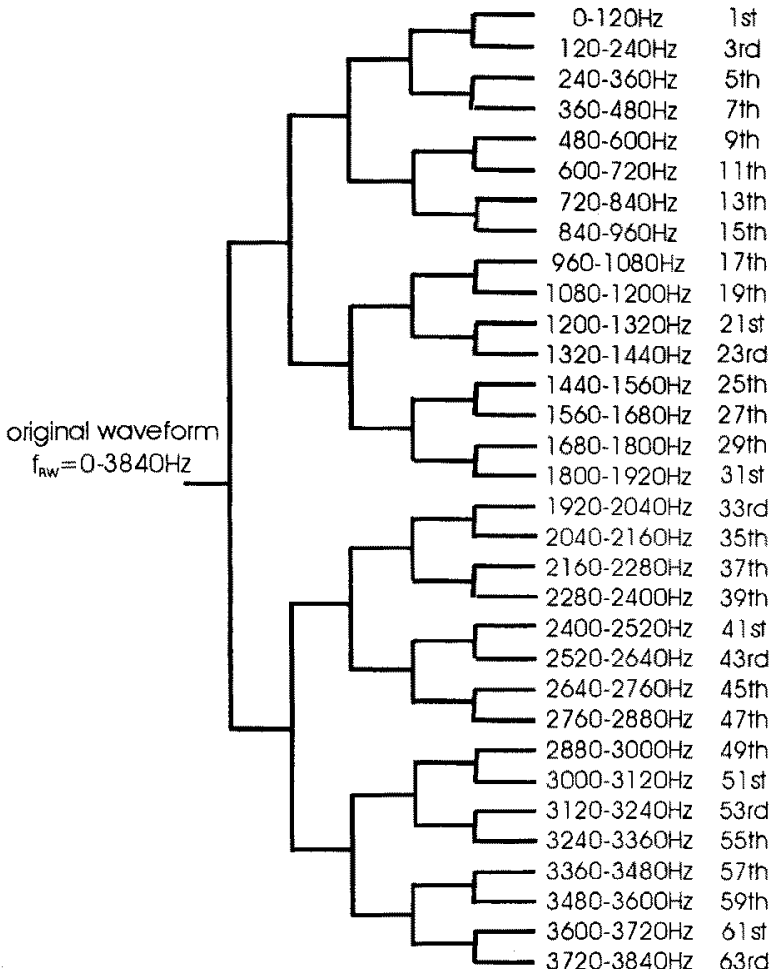

Fig. 4. Five-level wavelet packet decomposition scheme. Each tree represents the signal decomposition as depicted in Fig. 1. The frequency ranges of each band and the odd harmonic components included in each band are also given.

and thirteenth harmonics (odd integer harmonics), as follows (the unit of phases is in degree for simplicity):

$$
\begin{aligned}
v(t) & =\sqrt{2} \times\left[1.0 \sin (2 \pi 60 t)+0.2 \sin \left(2 \pi 180 t+135^{\circ}\right)\right. \\
& +0.2 \sin \left(2 \pi 300 t+150^{\circ}\right)+0.1 \sin \left(2 \pi 420 t+140^{\circ}\right) \\
& +0.08 \sin \left(2 \pi 540 t+40^{\circ}\right)+0.1 \sin \left(2 \pi 660 t+15^{\circ}\right) \\
& \left.+0.1 \sin \left(2 \pi 780 t+150^{\circ}\right)\right] \\
i(t) & =\sqrt{2} \times\left[1.0 \sin \left(2 \pi 60 t+10^{\circ}\right)+0.1 \sin \left(2 \pi 180 t+150^{\circ}\right)\right. \\
& +0.08 \sin \left(2 \pi 300 t+135^{\circ}\right)+0.08 \sin \left(2 \pi 420 t-22.5^{\circ}\right) \\
& +0.09 \sin \left(2 \pi 540 t+20^{\circ}\right)+0.07 \sin \left(2 \pi 660 t+45^{\circ}\right) \\
& \left.+0.08 \sin \left(2 \pi 780 t+120^{\circ}\right)\right] .
\end{aligned}
$$

As explained earlier, both voltage and current waveforms are decomposed up to 5 th level. Figure 6 shows the WTCs of voltage, current, and power at the selected bands. The WTCs of each band can be used to reconstruct the harmonic waveforms of each band using (6) and (7), and the result is shown in Fig. 7. Figure 7 demonstrates the time and frequency relationship, and it can be recognized that harmonics of both voltage and current as well as the power are stationary. Node 0 includes the fundamental frequency component, and the other nodes include the higher frequency components. The rms values of voltage and current of each band are computed from their WTCs at each band using (12) and (14), respectively. The square root of the sum of
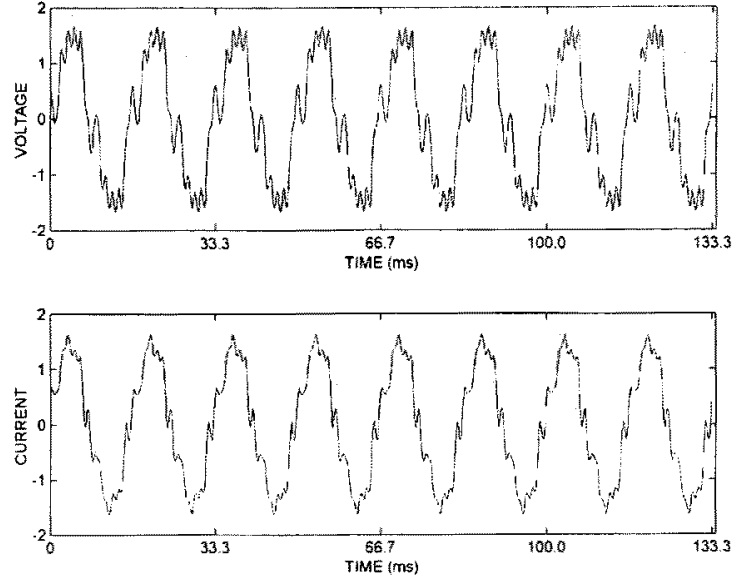

Fig. 5. Simulated voltage and current waveforms.
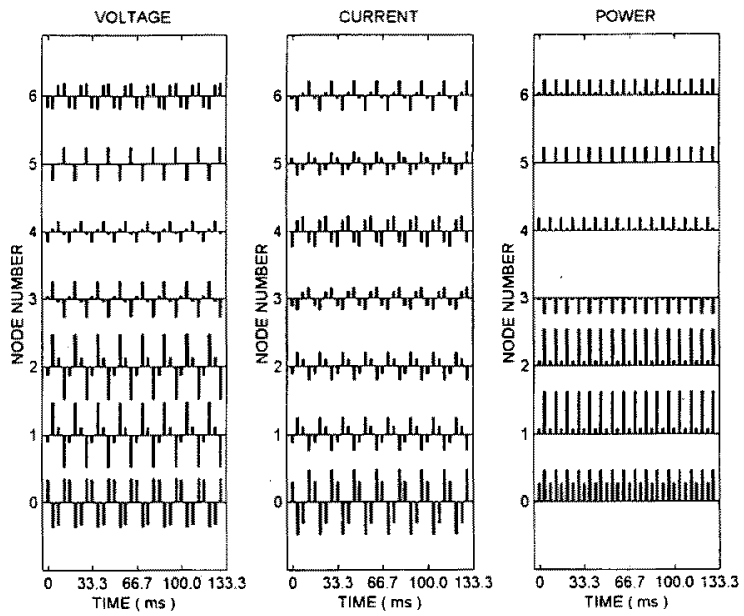

Fig. 6. The wavelet transform coefficients (WTCs) of simulated data for the first seven bands. (The ratio of node 0 and the other nodes is respectively $1: 5,1: 5$, and 1:50 for voltage, current, and power.)
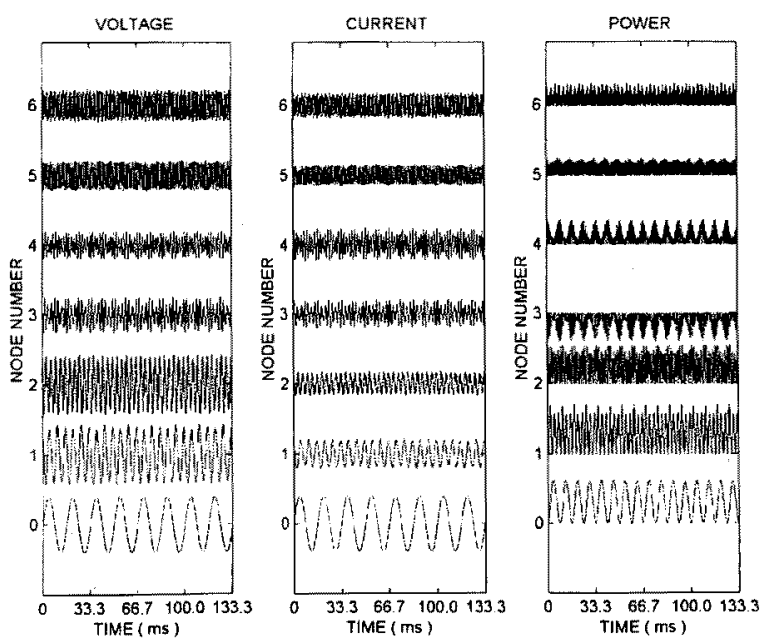

Fig. 7. Harmonic waveforms of each band reconstructed from the WTCs in Fig. 6.

rms values in all bands yields the total $\mathrm{rms}$ value of voltage or current (see Eqs. (12) and (14)). The WTCs of 
Table 1. Comparison between true and WPT for simulated data (rounded to 4 digits after comma).

\begin{tabular}{|c|c|c|c|c|c|c|c|c|}
\hline \multirow[t]{2}{*}{ Node } & \multirow{2}{*}{$\begin{array}{l}\text { Band } \\
(\mathrm{Hz})\end{array}$} & \multirow{2}{*}{$\begin{array}{l}\text { Band odd } \\
\text { Harmonics }\end{array}$} & \multicolumn{3}{|c|}{ TRUE } & \multicolumn{3}{|c|}{ WPT } \\
\hline & & & Vrms & Irms & Power & Vrms & Irms & Power \\
\hline 0 & $\mathrm{DC} \sim 120$ & 1st & 1.0000 & 1.0000 & 0.9848 & 1.0000 & 1.0000 & 0.9848 \\
\hline 1 & $120 \sim 240$ & $3 \mathrm{rd}$ & 0.2000 & 0.1000 & 0.0193 & 0.2003 & 0.1000 & 0.0193 \\
\hline 2 & $240 \sim 360$ & 5 th & 0.2000 & 0.0800 & 0.0155 & 0.1994 & 0.0797 & 0.0155 \\
\hline 3 & $360 \sim 480$ & 7 th & 0.1000 & 0.0800 & -0.0077 & 0.1002 & 0.0811 & -0.0076 \\
\hline 4 & $480 \sim 600$ & 9 th & 0.0800 & 0.0900 & 0.0067 & 0.0796 & 0.0891 & 0.0066 \\
\hline 5 & $600 \sim 720$ & 11th & 0.1000 & 0.0700 & 0.0061 & 0.0988 & 0.0690 & 0.0060 \\
\hline 6 & $720 \sim 840$ & 13th & 0.1000 & 0.0800 & 0.0069 & 0.1001 & 0.0799 & 0.0069 \\
\hline 7 & $840 \sim 960$ & 15 th & 0.0000 & 0.0000 & 0.0000 & 0.0001 & 0.0001 & 0.0000 \\
\hline & TOTAL & & 1.0566 & 1.0209 & 1.0316 & 1.0566 & 1.0209 & 1.0316 \\
\hline & error (\%) & & & & & 0.000000 & 0.000000 & 0.000000 \\
\hline
\end{tabular}

Table 2. Power factor and THDB for simulated data.

\begin{tabular}{c|rr}
\hline & TRUE & WPT \\
\hline p.f. & 0.9564 & 0.9564 \\
\hline THBDV(\%) & 32.2899 & 32.2815 \\
\hline THBDi(\%) & 20.1224 & 20.1222 \\
\hline
\end{tabular}

power are the products of the voltage and current WTC pairs registered at the same band and time, as written in (15). The power can be calculated using (17), and the energy is the power multiplied by the observation period $T$. Table 1 shows the true values and the results using the WPT approach with the associated node numbers, frequency bands, and odd harmonics which are included in these frequency bands. Here, the true values are derived analytically (or directly) from the simulation equations above. This table shows that the rms and power values of each band of the proposed approach closely match the true values. The results of total rms and total power is the same in all cases and no errors under the precision cited. This gives evidence that the rms and power calculations using the WPT approach, as written in (12), (14), and (17), are correct. The errors indicate the difference between the true values (which include total voltage rms, total current rms, and total power) and those obtained from the WPT approach. However, small leakage occurs to the calculation results in some frequency bands. These errors are due to non-ideal filter characteristics, since the filter pair has overlap spectral as seen in Fig. $3^{(1)(4)}$. Table 2 shows the power factor and THBD of voltage and current. This table shows that the WPT results closely match the true values or analytical results.

4.2 Analysis of Actual Waveform Data Figure 8 shows the voltage and current waveforms from the power supply $(100 \mathrm{~V})$ which is supplying several personal computers and other electronic devices in our laboratory. The bottom panel in this figure is the power waveform, that is the product of the $v(t)$ and $i(t)$ pair, from which the total average power in time domain can be computed.

Figure 9 shows the WTCs of voltage, current, and power at the first nine bands of interest. Figure 10 depicts the harmonic waveforms of each band. These waveforms are obtained from the reconstruction of WTCs in Fig. 9. The figure demonstrates the time and frequency relationship, and it can be recognized that harmonics of both voltage and current as well as the power are stationary. The rest of bands have very small or zero WTC amplitudes. This figure demonstrates the wavelet property of time-frequency localization. The
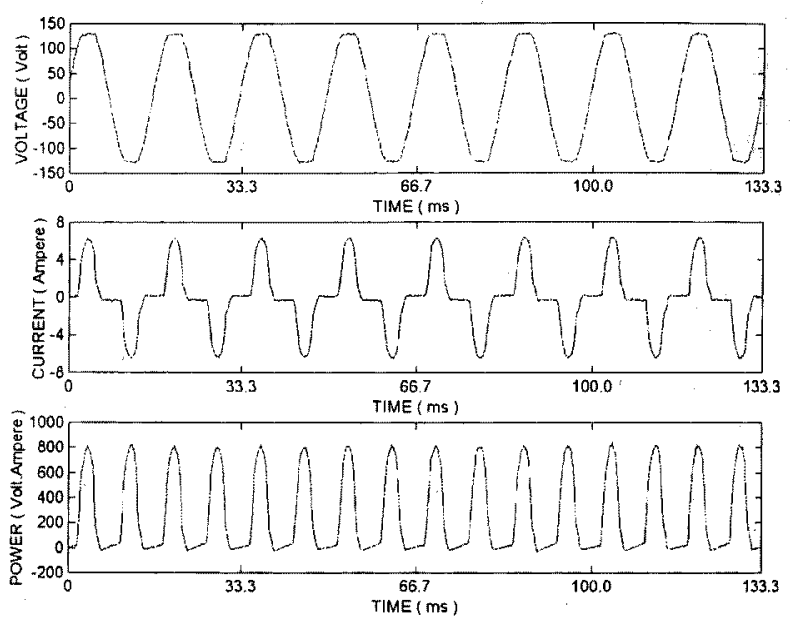

Fig. 8. Actual voltage and current waveforms. Power waveform is the product of voltage and current waveforms
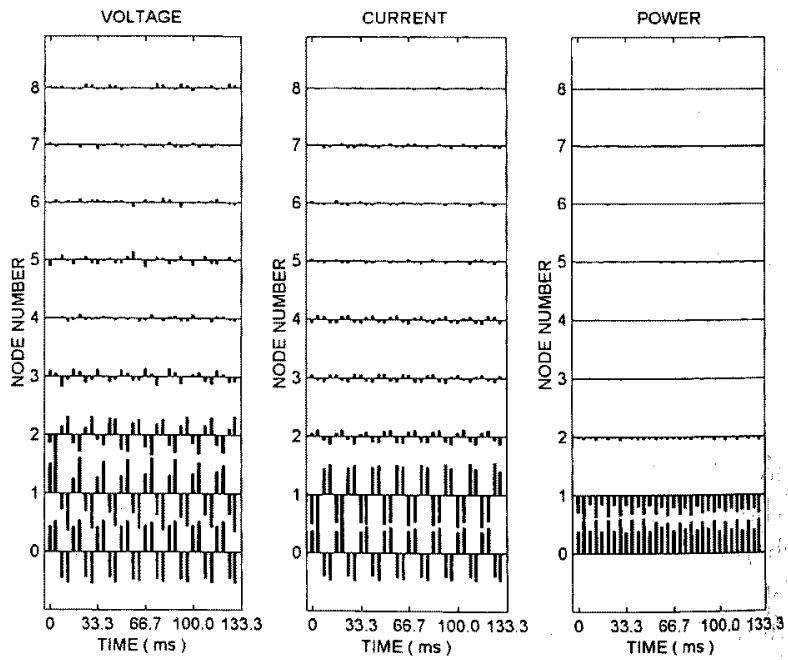

Fig. 9. The wavelet transform coefficients (WTCs) of actual data for the first nine bands of interest. (The ratio of node 0 and the other is respectively $1: 20,1: 2$, and 1:20 for voltage, current, and power.)

temporal variation of power WTCs correlates to the amplitude fluctuation of voltage current pair WTCs. 
Table 3. Comparison between FFT and WPT for actual data (rounded to 3 digits after comma).

The unit of voltage, current, and power is respectively volt, ampere, and watt.

\begin{tabular}{|c|c|c|c|c|c|c|c|c|}
\hline \multirow[t]{2}{*}{ Node } & \multirow{2}{*}{$\begin{array}{l}\text { Band } \\
(\mathrm{Hz})\end{array}$} & \multirow{2}{*}{$\begin{array}{l}\text { Band odd } \\
\text { Harmonics }\end{array}$} & \multicolumn{3}{|c|}{ FFT } & \multicolumn{3}{|c|}{ WP'T } \\
\hline & & & Vrms & Irms & Power & Vrms & Irmis & Power \\
\hline 0 & $\mathrm{DC} \sim 120$ & lst & 97.685 & 2,827 & 275.879 & 97.685 & 2.826 & 275.887 \\
\hline 1 & $120 \sim 240$ & $3 \mathrm{rd}$ & 4.825 & 1.636 & -7.477 & 4.817 & 1.644 & -7.553 \\
\hline 2 & $240 \sim 360$ & 5 th & 2.418 & 0.370 & -0.884 & 2.421 & 0.362 & -0.876 \\
\hline 3 & $360 \sim 480$ & 7 th & 0.939 & 0.193 & 0.130 & 0.911 & 0.194 & 0.104 \\
\hline 4 & $480 \sim 600$ & 9 th & 0.302 & 0.202 & -0.031 & 0.315 & 0.201 & -0.027 \\
\hline 5 & $600 \sim 720$ & 11 th & 0.611 & 0.070 & -0.036 & 0.601 & 0.073 & -0.038 \\
\hline 6 & $720 \sim 840$ & 13 th & 0.376 & 0.076 & 0.015 & 0.368 & 0.077 & 0.014 \\
\hline 7 & $840 \sim 960$ & $15 \mathrm{th}$ & 0.319 & 0.088 & -0.014 & 0.264 & 0.087 & -0.010 \\
\hline 8 & $960 \sim 1080$ & 17th & 0.303 & 0.029 & 0.000 & 0.362 & 0.033 & 0.005 \\
\hline & TOTAL & & 97.850 & 3.302 & 267.512 & 97.849 & 3.301 & 267.597 \\
\hline & $\operatorname{error}(\%)$ & & -0.000386 & -0.024575 & 0.031666 & 0.000000 & 0.000000 & 0.000000 \\
\hline
\end{tabular}

Table 4. Power factor and THDB for actual data

\begin{tabular}{c|rr}
\hline & FFT & WPT \\
\hline p.f. & 0.8279 & 0.8284 \\
\hline THBDv(\%) & 5.8186 & 5.7908 \\
\hline THBDi(\%) & 51.7046 & 51.7222 \\
\hline
\end{tabular}
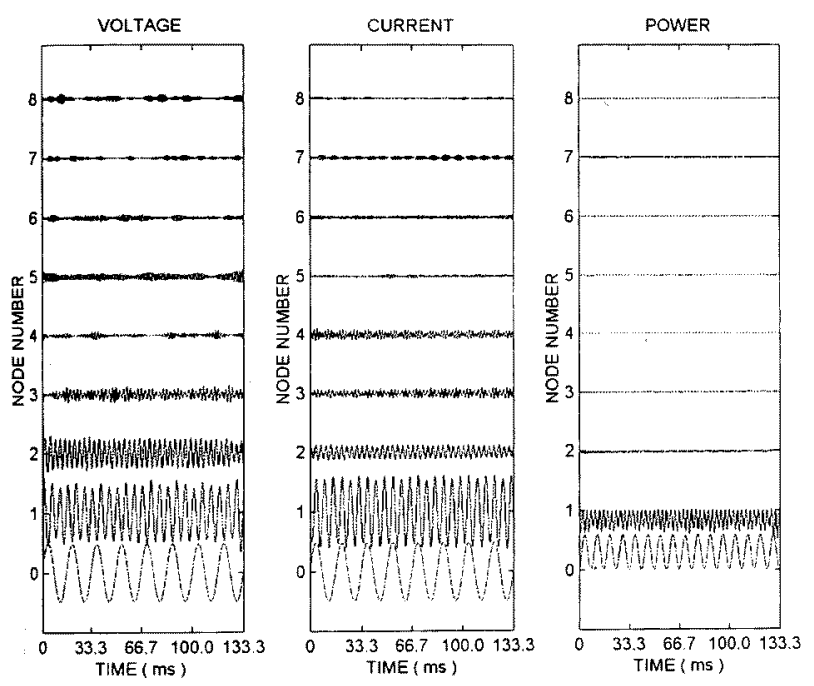

Fig. 10. Harmonic waveforms of each band reconstructed from the WTCs in Fig. 9.

Table 3 compares the results between the FFT values and the ones using the WPT approach. Power at each frequency of the FFT method can be computed based on magnitudes and angles resulting from the FFT analysis of voltage and current data. The table shows that the rms and power values of each band obtained by the two methods differ only slightly. The total results of rms and power of the proposed approach are almost the same in all cases. However, the relative errors indicate that the proposed approach provides the same total rms and total power values as the time domain reference under the precision cited, meanwhile the FFT method provides small errors. Here, the errors indicate the difference between the results of each technique and the results of time domain calculation (the time domain results are set as the reference). The results of total rms of voltage and current and total power in time domain are $97.849 \mathrm{~V}, 3.301 \mathrm{~A}$, and $267.597 \mathrm{~W}$, respectively, and the results are exactly the same as those obtained from the WPT method. The total rms of voltage and current in time domain is calculated using (11) and (13), respectively, while the total power in time domain is calculated using (16). The reason why the total $\mathrm{rms}$ and total power of the WPT approach equals to those obtained from the time domain is due to the wavelet properties of filter. The wavelet function $\psi(t)$ is a function with unit energy and zero mean (see Eq. (5)), so that if this wavelet function is convoluted with the original signal, then the energy of original signal which is distributed in the WTCs will remain constant ${ }^{(8)-(10)}$. From the Table 3 and Fig. 10, it can be seen that the power (or energy) is concentrated mainly at nodes 1 and 2 corresponding to the band of $\mathrm{DC}-240 \mathrm{~Hz}$, and only very small power distributed at the higher bands. Table 4 shows that the power factor and THBD values of the FFT and WPT methods are almost the same.

\section{Conclusions}

The WPT approach for rms and power/energy measurements has been proposed and its capability has been demonstrated. The evaluation was performed on two data: analytical and actual voltage current pair waveforms. Analytical data were used in the evaluation so the rms and power values of each frequency band and also the total rms and total power values could be compared with those obtained from analytical calculation. Actual power system data were used to evaluate the feasibility of this approach in the real condition, and the measurement results were compared with those obtained from the traditional frequency domain (FFT) method. The results of this WPT approach can be summarized as follows:

- The rms and power values of each frequency band derived from concurrent voltage and current data can be measured accurately under the precision cited. In the case of using simulated data, the results closely match to the analytical calculation results (or true values). The small differences in each band between the results of the WPT approach and true values are attributable to the non-ideal characteristics of the filter. In the case of using actual data, the results are closely match to the FFT calculation results. 
- Unlike the FFT method, the WPT approach provides total rms and total power which have the same values as the results from the time domain method.

- Besides resulting the distribution of $\mathrm{rms}$ and power/energy with respect to individual frequency bands, the bands also retain both the time and frequency relationship of the original instantaneous rms and power.

(Manuscript received June 6, 2001, revised October $25,2001)$

\section{References}

(1) Driesen, J., T.V. Craenenbroeck, R. Belmans, and G. Deconick, Electrical energy measurement in environments characterised by a detoriated power quality, IEE Ninth Int. Conf. on Metering and Tariffs for Energy Supply, 115-118, May 1999.

(2) Wilkinson, W.A., and M.D. Cox, Discrete wavelet analysis of power system transients,IEEE Trans. Power System, 10, 2039-2044, 1996.

(3) Robertson, D.C, O.I. Camps, J.S. Mayer, and W.B. Gish, Wavelets and electromagnetic power system transients, IEEE Trans. Power Delivery, 11, 1050-1058, 1996

(4) Yoon, W.K., and M.J. Devaney, Power measurement using the wavelet transform, IEEE Trans. Instrum. Meas., 47, 1205$1209,1998$.

(5) YuHua, G., and M.H.J. Bollen, Time-frequency and timescale domain analysis of voltage disturbances, IEEE Trans. Power Delivery, 15, 1279-1284, 2000.

(6) Santoso, S., W.M. Grady, E.J. Powers, J. Lamoree, and S.C Bhatt, Characterization of distribution power quality events with fourier and wavelet transforms, IEEE Trans. Power Delivery, 15, 247-254, 2000.

(7) Pham, V.L., and K.P. Wong, Wavelet transform-based algorithm for harmonic analysis of power system waveforms, IEE Proc. Gener. Transm. Distrib., 146, 249-255, 1999.

(8) Wickerhauser, M.V., Adapted wavelet analysis from theory to software, IEEE Press, New York, USA, 237-298, 1994.

(9) Mallat, S., A wavelet tour of signal processing, 2nd edition, Academic Press, San Diego, 1998.

(10) Beylkin, G., Wavelet, Multiresolution analysis and fast numerical algoritms, in INRIA lectures, 1-36, 1994.

Effrina Yanti Hamid (Non-member) She was born in In-

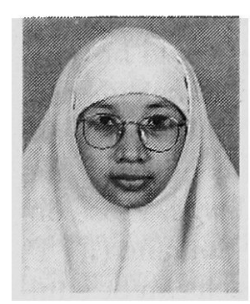
donesia in 1972. She received the bachelor and master degrees in electrical engineering from Institut Teknologi Bandung, Indonesia in 1995 and 1998, respectively. Now, she is finishing her Ph.D. thesis in the Department of Electrical Engineering, Osaka University. Her main interest is in the application of discrete wavelet packet transform to electric power system

Nobuaki Yokoyama (Student Member) He was born in Osaka, Japan in 1976. He received the bache-
lor degree in electrical engineering from Osaka
University in 2000. Now, he is a master course
student in the Department of Communication
Engineering, Osaka University. His main in-
terest is in harmonic analysis in electric power
system.
Zen-Ichiro Kawasaki (Member) He was born in Japan in

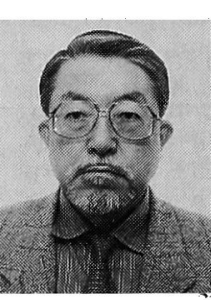
1949. He received the B.S., M.S. and Dr. Eng. degrees in communication engineering from Osaka University, Japan in 1973, 1975 and 1978, respectively. In 1989 , he joined the Department of Electrical Engineering, Osaka University. Currently, he is a Professor at the Department of Communication Engineering in the same university. His current research interests are in signal processing, diagnosis techniques of power apparatus, and the electromagnetic of lightning discharges. Dr. Zen-Ichiro Kawasaki is a member of IEEE, IEE of Japan, American Geophysical Union (AGU), and The Society of Atmospheric Electricity of Japan (SAEJ). 